\title{
TAKEOFF OF A FLYING MICROROBOT WITH COTS SENSOR PAYLOAD USING ELECTROHYDRODYNAMIC THRUST PRODUCED BY SUB-MILLIMETER CORONA DISCHARGE
}

Daniel S. Drew and Kristofer S. J. Pister

Berkeley Sensor and Actuator Center, UC Berkeley, USA

\begin{abstract}
This paper demonstrates the first flying microrobot using electrohydrodynamic thrusters, or ionocraft, to successfully take off while carrying an onboard commercial sensor package. The $13.6 \mathrm{mg}, 1.8 \mathrm{~cm}$ by $1.8 \mathrm{~cm}$ ionocraft is shown to take off while carrying a 40mg Flex PCB with 9-axis IMU and associated passives while tethered to a power supply. A new emitter electrode design has decreased corona onset voltage by over $30 \%$ and takeoff voltage by over $20 \%$ from previous efforts. Thrust density scaling with increasing numbers of emitter wires, continued geometric scaling for decreased operating voltage, device lifetime improvement via thin film deposition, and new assembly techniques are all explored.
\end{abstract}

\section{INTRODUCTION AND RELATED WORK}

Pico air vehicles, flying microrobots with mass under 100mg and characteristic length under $5 \mathrm{~cm}$, are being developed by a large number of research groups [1]. The majority of efforts focus on biomimetic flapping wing designs, typically using either piezoelectric or electromagnetic actuators. This work is unique in that it uses a microfabricated corona discharge based electrohydrodynamic actuator (Fig. 1) to produce thrust almost silently and with no moving parts, creating the highest thrust-toweight ratio pico air vehicle capable of takeoff (Fig. 2) to date [2].

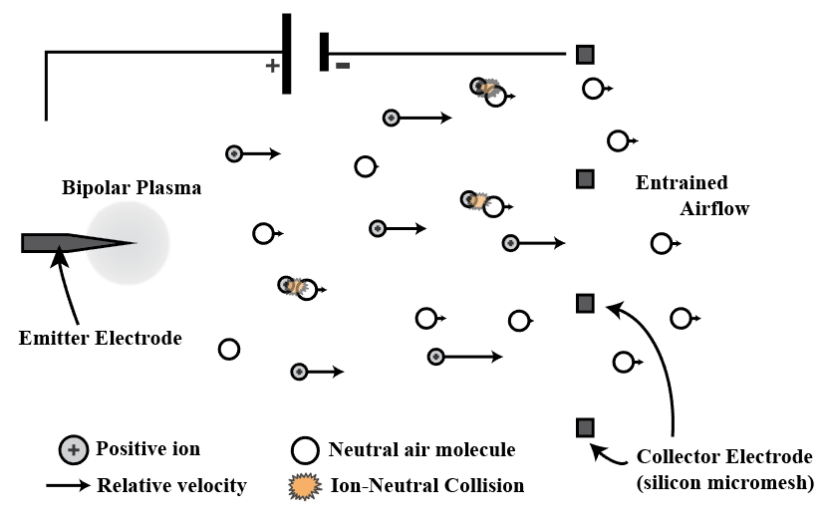

Figure 1: Thrust is produced when ions, drifting in the applied electric field, collide with neutral air molecules and impart momentum. Bipolar ions are generated in the corona plasma region localized at the sharp tip of the emitter electrode, but only positive ions (mainly $\mathrm{N}_{2}^{+}$) will drift towards the collector grid.

A one-dimensional model for electrohydrodynamic thrust, based on the electrostatic force on a volume of ions, leads to an expression for force in terms of ion drift current, $I$, distance the ions travel $d$, and the ion mobility, $\mu$, which is about $2 \mathrm{~cm}^{2} / \mathrm{Vs}$ for $\mathrm{N}_{2}^{+}$ ions in air [3]:

$$
F=\beta I d / \mu
$$

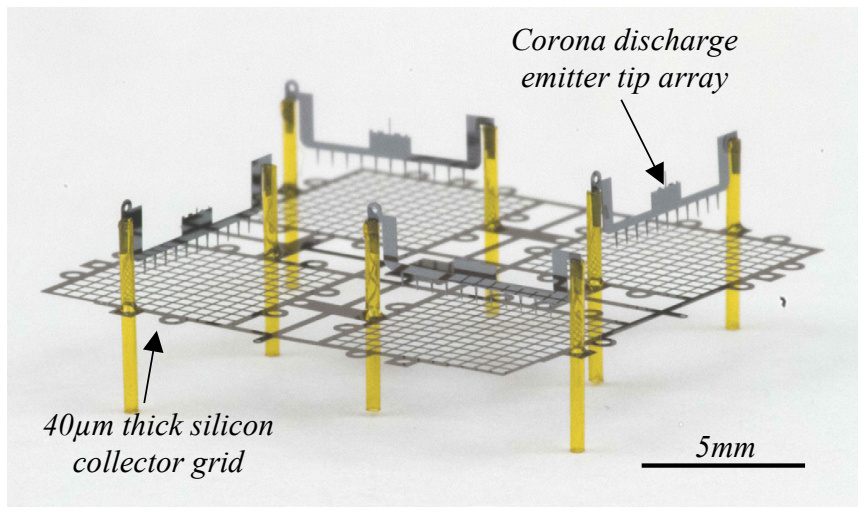

Figure 2: Photograph of the assembled ionocraft. With four individual addressable thrusters, it is about $1.8 \mathrm{~cm}$ by $1.8 \mathrm{~cm}$ and masses $13.6 \mathrm{mg}$. It is comprised of 13 individual components connected by a combination of mechanical slots and UV-curable epoxy. The design includes various tabs for handling and connection to power tethers.

The dimensionless term $\beta$ represents deviation from the ideal force caused by losses in the system due to factors like aerodynamic drag, bipolar plasma radius to drift distance ratio, and undesired ion path directions (e.g. horizontal velocity components "lost" as vertical thrust in collisions) [4].

Ions are generated using corona discharge, an atmosphericpressure, low-temperature DC plasma mechanism. In this process, an applied voltage between two asymmetric electrodes (e.g. a wire "emitter" and a plate "collector") yields a locally enhanced electric field around one of the two; if the potential is high enough, electrons will gain enough energy to initiate Townsend avalanche breakdown in the neutral air around the electrode, producing a bipolar plasma. Corona discharge is the most common mechanism of ion production for EHD force, with implementations using electrode gaps ranging from the 10 s of centimeters [5] to the sub-millimeter [6].

Although models for corona discharge in all but a few simple geometries are largely empirically derived, resultant ion current versus applied voltage generally follows the relationship:

$$
I=C V\left(V-V_{0}\right)
$$

Where $C$ is an empirical parameter that is a strong function of electrode geometry and $V_{0}$ is the corona onset voltage. The combination of these two governing equations yields an expression for EHD force that depends on two empirical factors, $C$ and $\beta$, that can be extracted from experimental data:

$$
F=\beta C V\left(V-V_{0}\right) d / \mu
$$

Designs for EHD thrusters can be assessed by attempting to maximize $C$ and $\beta$ while minimizing $\mathrm{V}_{0}$. Designs can further be 
assessed quantitatively by thrust-to-weight ratio and holistically by ease of fabrication and assembly.

The use of EHD thrust for a centimeter-scale robot capable of vertical takeoff has already been demonstrated; this work focuses on the steps being taken towards improving device performance, increasing assembly throughput and success rate, and incorporating useful payloads in an effort to move closer to controlled flight.

\section{EXPERIMENTAL METHODS}

Silicon electrodes with lithographically defined asperities ("tips") are fabricated in plane, fully released, and then rotated and assembled out of plane. Emitter wires with varying interelectrode gaps and asperity tip angles were fabricated. The fabrication process previously reported at MARSS 2017 [2] is adapted to a single mask SOI process in this work to increase device yield and decrease number of fabrication steps. An improved assembly method (Fig. 3) differs from prior work in that it: allows the electrodes to be designed for mechanical robustness (to survive assembly); separates the power tether connection point from the collector grid by a lithographically defined amount to prevent surface breakdown along the dielectric post; and allows the interelectrode gap to be controlled semi-lithographically.

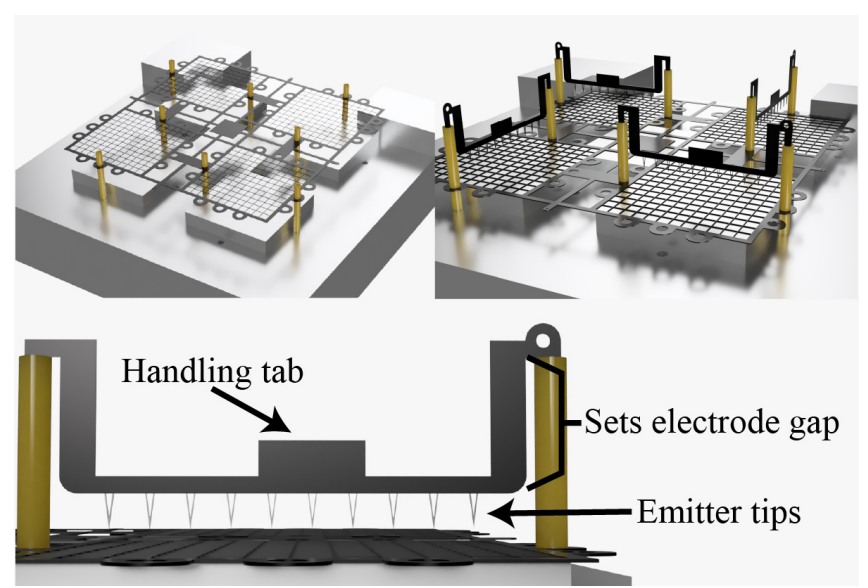

Figure 3: 3D model renders of the assembly process. Pre-cut silica tubes are inserted into slots in a CNC-milled aluminum jig. The ionocraft frame is slid down the guiding posts. The emitter wires are rotated out of plane and tapered guides are inserted into the silica tubes. The inter-electrode distance is now determined by a combination of lithography, slot depth, and tube length.

A Flex PCB board (Fig. 7 inset) has been fabricated containing a commercial 9-axis IMU, the InvenSense MPU-9250, as well as three associated passives and bonding pads. The board's $5 \mathrm{~mm}$ by $5.5 \mathrm{~mm}$ footprint allows it to be placed in a designated central region of the robot airframe without interfering with the surrounding thrusters. The total board mass of about $40 \mathrm{mg}$ is within the expected payload capacity reported in prior work.

To measure output force, devices are mounted on a threaded nylon post fixed directly to a Phidgets $100 \mathrm{~g}$ Micro load cell. For full quad-thruster ionocrafts there is a dedicated region in the center of the airframe for mounting; single thrusters are mounted using one of the unused silica tube slots along the grid frame. Multiple instruments are controlled via GPIB for synchronized force-currentvoltage sweeps.

\section{RESULTS AND DISCUSSION}

The presented data is from a range of fabricated devices; unless otherwise noted, collected data is from the first trial using each device to reduce the effect of lifetime performance degradation. Although presented data is first passed through a low pass filter in the Phidgets data hub and then averaged over 3 seconds of collection for each measurement, there was still significant noise $(\mathrm{SD}=35 \mu \mathrm{N}$, $\mathrm{n}=11$ ) in the strain sensor compared to the values found in this work.

\section{Electrode Geometry}

In a widely-used set of empirically derived equations for corona onset voltage formulated by Peek [8] for various electrode geometries, it is found that $V_{0} \propto \ln (d)$. Decreasing the electrode gap from $500 \mu \mathrm{m}$ to $250 \mu \mathrm{m}$ (Fig. 4) was shown to decrease the onset voltage, as found by the 0 -intercept of the curve fit to Eq.2, by approximately $400 \mathrm{~V}$, a larger change than predicted by this trend. Although related work already shows deviation from Peek's equations in sub-millimeter corona discharges [9], this greater deviation at even smaller gaps bears further investigation. The empirical factor $C$, a metric for change in ion current as a function of input voltage, was shown to increase by about $230 \%$; because current must double to produce the same output force at half the drift distance (Eq. 1), this would ideally correspond to a smaller required operating voltage swing for the same force production range.
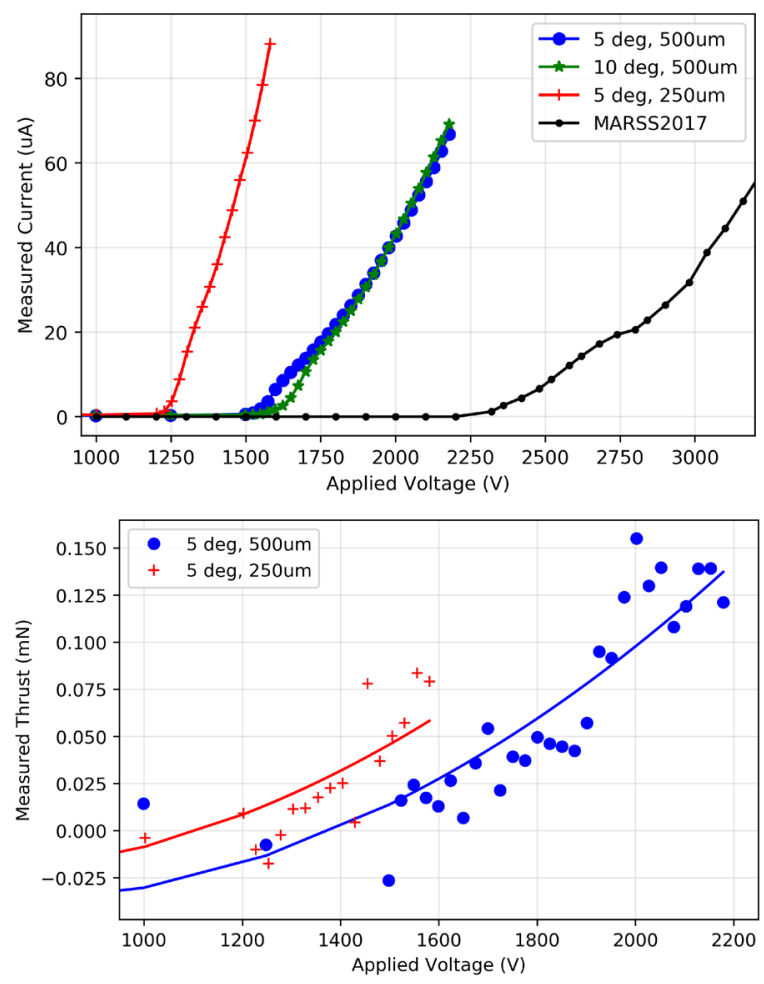

Figure 4: Current and force versus voltage curves for thrusters with different electrode gaps or different drawn emitter tip angles. The current-voltage data from MARSS2017 [2] is a $20 \mu \mathrm{m}$ by $40 \mu \mathrm{m}$ cross section silicon "wire" with no lithographically defined asperities at an electrode gap of $1 \mathrm{~mm}$. The $250 \mu \mathrm{m}$ electrode gap device uses a collector grid with $250 \mu \mathrm{m}$ spacing. The plotted lines in the forcevoltage plot are 2-degree polynomial fit lines (Eq. 3).

The $250 \mu \mathrm{m}$ gap device has an extracted $\beta$ via linear fit of current-force data of $0.33,44 \%$ lower than the extracted value of 0.59 for the $500 \mu \mathrm{m}$ gap, 5-degree device. Although the absolute value of this factor may be incorrect due to variation in ion mobility from the assumed $2 \mathrm{~cm}^{2} / \mathrm{Vs}$, the trend is expected from prior work 
[4] and likely largely due to the effect of aerodynamic drag on the smaller spacing of the $250 \mu \mathrm{m}$ collector grid. Further, device failure (i.e. arcing) occurred before the same maximum force could be produced by the smaller electrode gap, indicating that emitter tip radius, $r$, should be decreased in an effort to increase the $d / r$ ratio critical for corona discharge.

The onset voltage was not found to change significantly (about $50 \mathrm{~V}$ ) between 5-degree and 10-degree drawn emitter tip angle wires. This could indicate some limit in dimensional scaling following DRIE and current lithography methods, as asperity drawn angle was shown to have a strong effect on onset voltage in prior work [4].

\section{Thrust Density}

Adding more emitter wires per collector grid should have the effect of increasing both $C$ and thrust-to-weight ratio at the cost of additional assembly time. Experiments show that corona discharge current scales approximately linearly with number of emitter wires (Fig. 5) at a wire spacing of $3 \mathrm{~mm}$ for the 2 -wire case and $1.5 \mathrm{~mm}$ for the 3 -wire case. Going from one emitter wire per collector grid to three increases $C$ by about $250 \%$, while $\beta$ decreases from 0.59 to 0.53 . The fact that current did not scale linearly may be attributable to assembly or device degradation nonuniformity, and the decrease in beta is within the measurement error for the strain sensor used in this work.
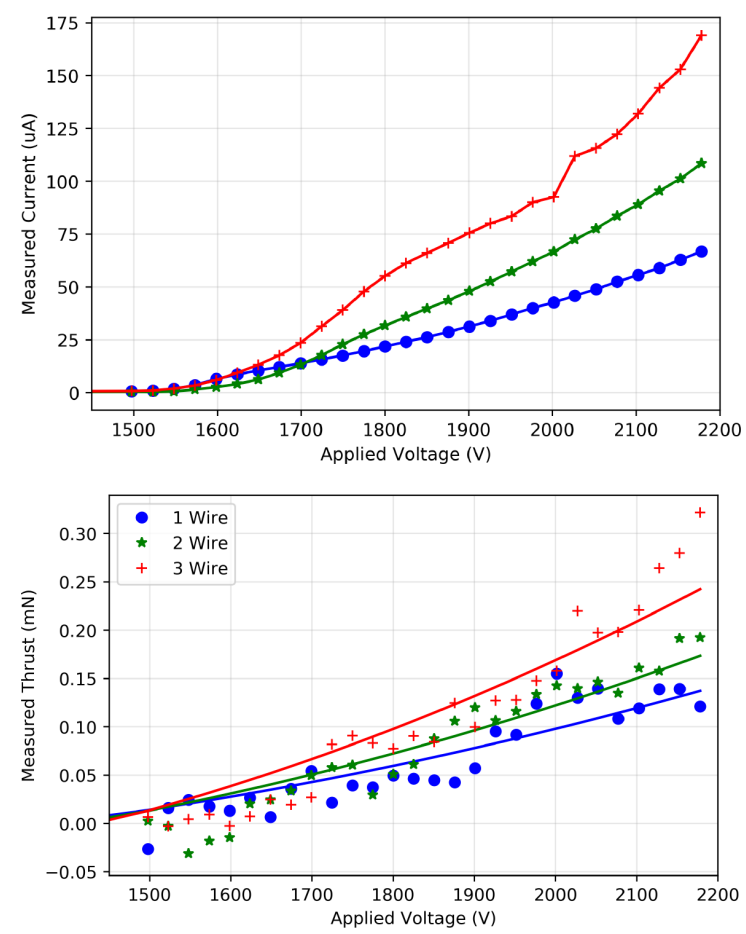

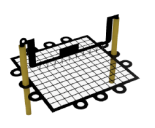

-1 Wire-

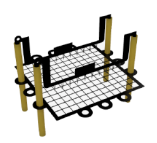

-2 Wires-

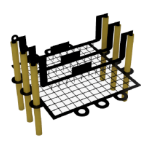

-3 Wires=
Figure 5: Current-voltage and force-voltage curves produced by varying the number of emitter wires in a thruster. Tests are for 5degree emitter tips, an electrode gap of $500 \mu \mathrm{m}$, and a collector grid spacing of $500 \mu \mathrm{m}$. The plotted lines in the force-voltage plot are 2degree polynomial fit lines.

The mass of an additional emitter wire and two silica posts is only $1.5 \mathrm{mg}$ while the maximum thrust is increased by approximately
$100 \mu \mathrm{N}(10 \mathrm{mg})$ per set, showing a large potential boost to thrust-toweight ratio. Prior work in FEA simulation indicates that the space charge region developed from a single emitter wire's corona discharge spans a horizontal radius approximately equal to the electrode gap distance. This indicates that wire neighbor spacing can be decreased from $1.5 \mathrm{~mm}$ to $1 \mathrm{~mm}$ and the maximum number of emitter wires per collector grid could double to 6 without significant neighbor interactions; although this would certainly continue to provide an aerial thrust density and thrust-to-weight ratio benefit, assembly complexity would be prohibitive with the current design.

\section{Device Lifetime}

Performance of a corona discharge based EHD thruster can be expected to degrade over time, largely as a result of interactions of the bipolar plasma region with the emitter electrode. Initial experiments saw logarithmic decay $\left(\mathrm{R}^{2}=0.98\right.$ fit $)$ of discharge current over time with a constant applied voltage (Fig. 6). Scanning electron micrographs showed large amount of physical damage to the emitter wires, localized to the tips themselves, with a damage radius of around $60 \mu \mathrm{m}$. With the hypothesis that this damage is a result of energetic ion ablation in the bipolar plasma (a plasma radius of $50 \mu \mathrm{m}$ is often assumed in literature), the tips were sputtered with a $100 \mathrm{~nm}$ titanium nitride layer, turned over, and sputtered with another $100 \mathrm{~nm}$ layer. Subsequent testing showed no degradation of performance in the same time period. It is expected that the new failure mode will be through oxidation of the tips enhanced by the energetic oxygen in the bipolar plasma; TiN has been experimentally shown to grow $10 \mathrm{~nm}$ thick oxide films after 1000 seconds in a $200^{\circ} \mathrm{C} \mathrm{O}_{2}$ plasma asher [9].
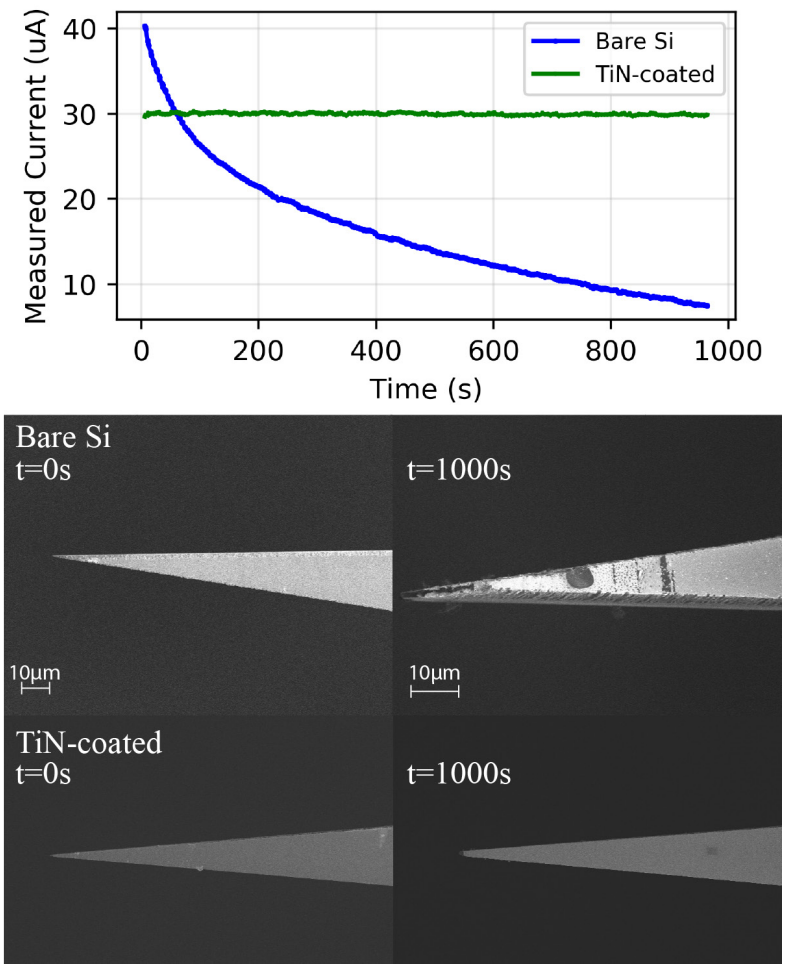

Figure 6: Device lifetime quantified by measured ion current versus time at a constant operating voltage. Bare silicon emitter wires saw immediate performance degradation during testing, while wires coated by about 100nm of sputtered TiN had stable performance up to the measured 1000s of operation. Devices are 10-degree tips at a $500 \mu \mathrm{m}$ gap with $1800 \mathrm{~V}$ applied. 


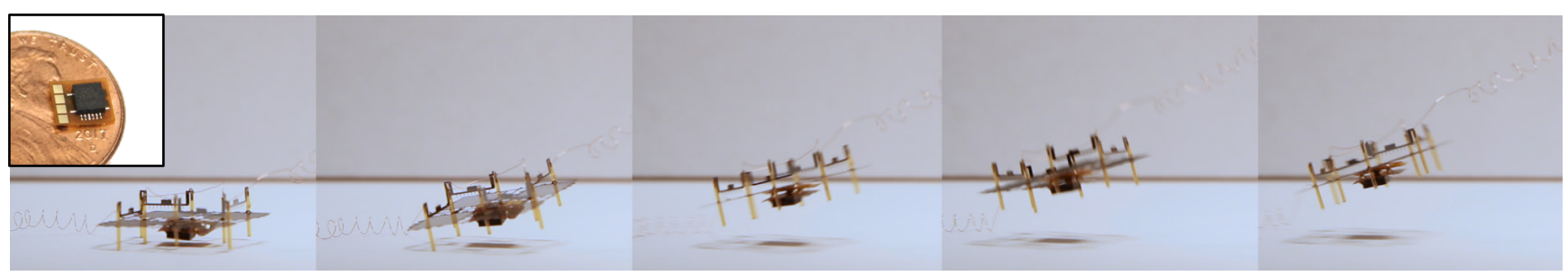

Figure 7: An ionocraft with assembled 40mg Flex PCB IMU board attached to the airframe center pad. At about 2100V, the ionocraft is able to take off from the table; the power and ground wires prevent the ionocraft from going any higher. There is only one high voltage line attached here, with connections between the emitter wires done on the ionocraft itself. The IMU board is not wired or active during takeoff. Inset: Flex PCB IMU board on a U.S. penny. The board is about $5 \mathrm{~mm}$ by $5.5 \mathrm{~mm}$ and masses $40 \mathrm{mg}$.

\section{Takeoff with Sensor Payload}

Current flying microrobots are typically payload constrained; either they fail to lift the weight of their own actuators and airframe, or must seek to develop novel sensing schemes and platforms to optimize for mass. The relatively high thrust-to-weight ratio (about 4.5 in this work) of the ionocraft means that it may be possible to use standard commercial sensors, simplifying development and implementation. To prove the feasibility of future controlled flight using a commercial sensing package we developed a 40mg FlexPCB with 9-axis IMU, attached it to a designated central region in the ionocraft airframe with UV-curable epoxy, and made the robot take off (Fig. 7). This single-emitter design had an unladen takeoff voltage of about $2000 \mathrm{~V}$ (2100V with IMU); this number should decrease by using multiple emitter wires per thruster, but with the assembly jig used in this work it was difficult to reliably assembly such a version.

\section{CONCLUSIONS AND FUTURE WORK}

Although we have validated the use of multiple emitter wires as a way to increase device performance, with the current design each new emitter wire requires both its own pair of silica posts (with associated epoxy) and electrical connection to its neighbors, decreasing benefit to thrust-to-weight ratio. Future work will explore new methods, for example designing mechanical interlocks into the components themselves, to overcome these challenges.

Future efforts will focus on further decreasing operating voltage and bringing aerial thrust density closer to theoretical limits. Work using FEA simulation will investigate the potential for taking advantage of space charge region interaction between neighboring wires to increase flow efficiency (e.g. through induced jet flow). We also see potential for decreasing aerodynamic drag on the collector grid without influencing corona discharge behavior by using nonuniform geometries (e.g. a grid spacing that varies based on proximity to emitter tip).

Efficiency is a key concern when developing millimeter-scale robots, where resource constraints are projected to dominate potential function. Thrust efficiency in terms of $N / W$ for an EHD thruster scales $\propto 1 / E_{\text {drift }}$. As electrode gap continues to decrease in an effort to decrease operating voltage, the effect of decreasing electrode $d / r$ ratio on minimum drift field strength, and therefore efficiency, becomes more pronounced. The devices in this work have efficiencies that range from $2 \mathrm{mN} / \mathrm{W}$ to $1 \mathrm{mN} / \mathrm{W}$ over their operating range, unacceptably low for a truly autonomous flying robot with any useful mission time. It may be necessary to increase emitter tip sharpness beyond what is possible with standard lithographic techniques, for example with thermal oxide sharpening, in order to operate ionocraft in higher efficiency regimes by trading off output force.

The significant performance degradation of the bare silicon emitter wires shown in Fig. 6 implies that the IV and FV curves presented in this work are strongly (negatively) affected during their approximately 90 second measurement period. Although assuming uniform degradation for all measured emitter wires allows us to draw conclusions about the same general trends, absolute values (e.g. $C, \beta$ ) will be subject to change in future work even for emitter tips with nominally identical radii.

In summary, a steady continued development of the quadthruster ionocraft has put controlled, tethered flight within reach. Work will continue on decreasing operating voltage while increasing thrust density in order to feasibly include a high voltage boost conversion circuit, similar to [10], and microprocessor onboard.

\section{REFERENCES}

[1] R. J. Wood, B. Finio, M. Karpelson, K. Ma, N. O. PérezArancibia, P. S. Sreetharan, H. Tanaka, and J. P. Whitney, "Progress on 'pico'air vehicles," Int. J. Rob. Res., vol. 31, no. 11, pp. 1292-1302, 2012.

[2] D. S. Drew and K. S. J. Pister, "First takeoff of a flying microrobot with no moving parts," in Manipulation, Automation and Robotics at Small Scales (MARSS), 2017 International Conference on, 2017, pp. 1-5.

[3] L. Pekker and M. Young, "Model of ideal electrohydrodynamic thruster," J. Propuls. Power, vol. 27, no. 4, pp. 786-792, 2011.

[4] D. S. Drew and K. S. J. Pister, "Geometric optimization of microfabricated silicon electrodes for corona discharge-based electrohydrodynamic thrusters," Micromachines, vol. 8, no. 5, p. 141, 2017.

[5] K. Masuyama and S. R. H. Barrett, "On the performance of electrohydrodynamic propulsion," Proc. R. Soc. A Math. Phys. Eng. Sci., vol. 469, no. 2154, pp. 20120623-20120623, 2013.

[6] A. O. Ong, A. R. Abramson, and N. C. Tien, "Electrohydrodynamic microfabricated ionic wind pumps for thermal management applications," J. Heat Transfer, vol. 136, no. 6 , p. $61703,2014$.

[7] R. Tirumala, Y. Li, D. A. Pohlman, and D. B. Go, "Corona discharges in sub-millimeter electrode gaps," J. Electrostat., vol. 69, no. 1, pp. 36-42, 2011.

[8] F. W. Peek, Dielectric phenomena in high voltage engineering. New York: McGraw-Hill, 1915.

[9] H. G. Tompkins and J. A. Sellers, "Oxidation of TiN in an oxygen plasma asher," J. Vac. Sci. Technol. A Vacuum, Surfaces, Film., vol. 12, no. 4, pp. 2446-2450, 1994.

[10] M. Karpelson, G.-Y. Wei, and R. J. Wood, "Milligram-scale high-voltage power electronics for piezoelectric microrobots," in Robotics and Automation, 2009. ICRA'09. IEEE International Conference on, 2009, pp. 2217-2224.

\section{CONTACT}

D. S. Drew; ddrew73@berkeley.edu 